\title{
Anti-sulfatide reactivity in patients with celiac disease
}

\author{
Domenica Saccomanno ${ }^{a}$, Carolina Tomba ${ }^{b}$, Francesca Magri ${ }^{a}$, Philippe Backelandt ${ }^{c}$, Leda Roncoroni ${ }^{b, d}$, \\ Luisa Doneda ${ }^{d}$, Maria Teresa Bardella ${ }^{b}$, Giacomo Pietro Comi ${ }^{a}$, Nereo Bresolin ${ }^{a}$, Dario Conte ${ }^{b}$ and Luca Elli ${ }^{b}$ \\ 'Department of Pathophysiology and Transplantation, Neurology Unit, Dino Ferrari Center, University of Milan, Fondazione IRCCS Ca' Granda- \\ Ospedale Maggiore Policlinico, Milan, Italy; ${ }^{\mathrm{b} C e n t r e}$ for the Prevention and Diagnosis of Celiac Disease, Gastroenterology and Endoscopy \\ Unit, Fondazione IRCCS Ca' Granda-Ospedale Maggiore Policlinico, Milan, Italy; ' ZenTech S. A., Angleur, Belgium; 'Department of Biomedical, \\ Surgical and Dental Sciences, University of Milan, Milan, Italy
}

\begin{abstract}
Objective: To explore a possible significance of the presence of anti-ganglioside and anti-sulfatide anti- bodies in sera of adult patients with celiac disease (CD) in different clinical scenario.

Methods: We selected 22 adult patients with newly diagnosed CD and 20 age-sex matched non-CD controls. Patients' serum was tested - before and after at least 6 months on a gluten-free diet (GFD) - for anti-GM1, GM2, GM3, GD1a, GD1b, GD3, GT1a, GT1b, GQ1b and sulfatide IgM, IgG and IgA auto- antibodies, by means of a dot blot technique and enzyme-linked immunosorbent assay (ELISA).

Results: We found the presence of auto-antibodies in untreated patients. In particular, anti-sulfatide IgG antibodies were present in $8(36 \%)$ patients independently of the presence of neurological symp- toms. Anti-sulfatide IgA antibodies were present in 3 (19\%) patients. During GFD, anti-sulfatide IgG dis- appeared in all the patients, whereas IgA were observed in 2 patients. Anti-sulfatide, antiGM1 and anti-GM2 IgM antibodies were also observed in 2 patients on a GFD. All the other auto-antibodies were absent and no demographic or clinical parameters were associated. Non-CD controls did not pre- sent any auto-antibody.

Conclusions: We found anti-sulfatide IgG antibodies in CD patients on a gluten-containing diet. Anti-sulfatide IgA antibodies persisted during GFD together with the occurrence of other IgM auto- antibodies. These data suggest a possible link between gluten and IgG auto-antibodies.
\end{abstract}

\section{Introduction}

Celiac disease $(C D)$ is a common autoimmune enteropathy triggered by the ingestion of gluten proteins in genetically susceptible individuals carrying the HLA type II DQ2 and/or DQ8 haplotypes. Although the small bowel is the main targeted organ and localization of the inflammatory damage, $C D$ is considered a systemic disorder as different tissues and organs, including the central and peripheral nervous systems, are involved. Approximately, $10 \%$ of CD patients show neurological symptoms,[1] in particular cerebellar ataxia, peripheral neuropathy (PN) and epilepsy.[2-4]

The pathogenesis of the neurological complications in $C D$ is still unclear; vitamin deficiency could be considered in case of malabsorption and extensive small-bowel atrophy. However, this mechanism does not justify all the cases, especially those with normal levels of vitamins and without a clear malabsorptive syndrome. It could be supposed that systemic inflammation and the consequent increase in cytokines and auto-antibodies levels would lead to an immune reaction against nervous system components. Moreover, the response of neurological symptoms to a gluten-free diet (GFD) remains controversial. From this point of view, if neurological symptoms are independent of GFD, the presence of a genetic background facilitating the onset of multiple autoimmune diseases (including neurological autoimmune disorders) with CD may represent a co-factor.[5]

The presence of anti-ganglioside antibodies in the serum of $C D$ patients may explain neurological symptoms in $C D$, as recently supposed by different researchers; in fact, IgG antibodies against gangliosides have been found in the sera of adult CD patients with PN.[6]

Gangliosides are glycosphingolipids (GM1, GM2, GM3, GD1a, GD1b, GD3, GT1a, GT1b, GQ1b) which contain sialic acid and are present in large amounts in the nervous system, especially in myelin. They can be antigenic targets in a variety of autoimmune neuropathies. The identification of antiganglioside antibodies in large cohorts of patients with a wide range of acute and chronic peripheral neuropathies and their association with particular clinical phenotypes have been reported.[7] The possible involvement of anti-sulfatide antibodies in CD remains unknown. Sulfatide is a common glycolipid in the peripheral nerve myelin and dorsal root ganglia [8] and may be targeted by IgM auto-antibodies associated with the onset of sensory axonal neuropathy or predominantly demyelinating sensorimotor neuropathy.[9]

Others authors confirmed the presence of anti-ganglioside IgG antibodies in CD patients but did not find any correlation 
with neurological symptoms, suggesting that anti-ganglioside antibodies are an epiphenomenon due to some abnormal antigenic presentation during an inflammatory process.[10]

Tursi et al. [11] demonstrated that the majority of neurological symptoms remain unmodified after GFD. Other researchers found that about $50 \%$ of the $C D$ patients with neurological symptoms, resulting positive for at least one of the anti-ganglioside IgM or IgG antibodies, shows an antibody disappearance after 1 year on GFD.[12]

The present scenario appears conflicting and contradictory and nothing is known about the possible presence of antiganglioside and anti-sulfatide IgA antibodies, theoretically produced by the inflamed duodenal mucosa.

The aim of our study was to explore a possible significance of the presence of anti-ganglioside and anti-sulfatide immunoglobulins in the sera of $C D$ patients.

\section{Methods}

\section{Protocol and participants}

The sera of $C D$ patients attending the Center for the prevention and diagnosis of $\mathrm{CD}$ at the Fondazione IRCCS Ca' Granda Ospedale Maggiore Policlinico were collected before and after at least 6 months on a strict gluten-free dietary regimen. $C D$ diagnosis was based upon the presence of antitransglutaminase type II antibodies and duodenal atrophy (Marsh 3).[13] Only the sera of patients showing the normalization of anti-transglutaminase and anti-gliadin antibodies during GFD were analyzed. The sera of healthy blood donors (HBD) were also analyzed as non-CD controls. The CD clinical characteristics (classical $C D$, non-classical) were defined following the Oslo definitions.[14] Any neurological disorders were defined based on clinical neurological examinations and instrumental investigations, such as electrophysiological testing and encephalic magnetic resonance imaging. The study was approved by the local ethics committee of the Fondazione IRCCS Ca' Granda Ospedale Maggiore Policlinico, Milano (protocol no. 850).

\section{Anti-ganglioside and anti-sulfatide IgG, IgM and IgA dosage}

The presence of anti-gangliosides (GM1, GM2, GM3, GD1a, GD1b, GD3, GT1a, GT1b, GQ1b) in the sera of CD patients, before and after GFD, and non-CD controls was detected by means of the dot blot technique (DOTZEN", Zentech, Angleur, Belgium). The manufacturer's instructions were followed. Briefly, the sera were incubated for $2 \mathrm{~h}$ at $4{ }^{\circ} \mathrm{C}$ on a rocking shaker, washed three times and further incubated with peroxidase-conjugated anti-human immunoglobulins and a substrate solution revealing the presence of IgG, IgM or IgA auto-antibodies. The presence of blue-colored spots with various intensity was considered positive.

Peroxidase-conjugated anti-human IgA was produced ex novo from Zentech. Goat anti-human IgA-HRP was diluted into a phosphate buffer containing $\mathrm{NaCl}$ and calf serum.

The sera positive at dot blot analysis underwent a confirmatory analysis dosage by means of enzyme-linked immunosorbent assay (ELISA), following a previously reported procedure.[9] This method is used in the routine diagnostic for anti-ganglioside antibodies, according to the criteria of the Italian Association of Neuro-Immunology (AINI) of 2004.[15]

The presence of anti-sulfatide IgM, IgG and IgA auto-antibodies in the sera of CD patients, before and after GFD, and nonCD controls was also detected by ELISA.

Gangliosides (Hytest, Turku, Finland) and sulfatide (Sigma Aldrich, St. Louis, MO) were added in ethanol to each well of microplate (NUNC-Immuno ${ }^{\text {TM }}$ Plates, Polysorb U96, VWR, Radnor, Pennsylvania) until complete evaporation at $4{ }^{\circ} \mathrm{C}$. After the saturation of un-reactive sites with a blocking solution for $4 \mathrm{~h}$, a patient's serum was added in duplicate to the wells and incubated overnight. After five washes, IgM, IgG or IgA binding was detected with peroxidase-conjugated rabbit anti-human IgM, IgG and IgA, respectively (Dako, Glonstrup, Denmark), diluted 1:500 in a blocking solution, for $2 \mathrm{~h}$. After five washes, the color reaction was developed for $1 \mathrm{~h}$ before adding $0.1 \mathrm{M} \mathrm{H}_{2} \mathrm{SO}_{4}$ and absorbance measured at $492 \mathrm{~nm}$. The sera were considered positive when the difference of the optical density (OD) between glycolipids-coated wells and BSA-coated wells exceeded 0.05 OD units, in both duplicate wells. Positive sera were titrated by a serial two-fold dilution until negative.

No anti-sulfatide reactivity IgG and IgA was found in nonCD controls at a dilution of 1:16,000 and this dilution was used to analyze the sera of the patients recruited in our study. The samples were considered to be positive for antisulfatide IgG and IgA antibodies when titers were equal or greater than $1: 16,000$.

\section{Standardization ELISA}

Enzyme immunoassay with highly purified gangliosides is commonly used from different services for the routine diagnostic of the anti-gangliosides antibodies in sera of patients affected by dysimmune neuropathies, according to the criteria of the Italian Association of Neuro-Immunology (AINI) of 2004.[15]

Anti-ganglioside and sulfatides IgM antibodies are analyzed in the serum of patients with chronic dysimmune neuropathies while anti-ganglioside IgG antibodies for acute clinical phenotype.

All serum samples are added in duplicate to the initial dilution of 1:640, while for anti-sulfatide antibodies a dilution of 1:8000 is used. Controls of positive sera of all tested gangliosides are included in each experiment to ensure validity and reproducibility.

\section{Statistical analysis}

All the assumptions were verified by software, i.e., GraphPad Prism version 5.0 (GraphPad Software, La Jolla, CA), and a $p$ value $<0.05$ two tails was considered as statistically significant. Kolmogorov-Smirnov's test was used to assess the normal distribution of the data. Continuous variables were analyzed by the ANOVA one-way variance test. The 
Table 1. Demographic characteristics of the studied cohort.

\begin{tabular}{|c|c|c|c|}
\hline & $\mathrm{CD} \mathrm{N} 1 / 422$ & Non-CD N 1/4 20 & $p$ \\
\hline \multicolumn{4}{|l|}{ Gender } \\
\hline Female, $n(\%)$ & $17(78)$ & $15(75)$ & NS \\
\hline Male, $n(\%)$ & $5(23)$ & $5(25)$ & \\
\hline Age at diagnosis (years) & $40(27-64)$ & $39(26-65)^{*}$ & NS \\
\hline GFD duration (months) & 21 & NA & \\
\hline \multicolumn{4}{|l|}{ Type of presentation } \\
\hline Classical CD & $11(50)$ & NA & \\
\hline Non classical CD & $11(50)$ & & \\
\hline
\end{tabular}

Table 2. Characteristics of patients with neurological symptoms.

\begin{tabular}{|c|c|c|c|c|}
\hline \multirow[b]{2}{*}{ Patient } & \multirow{2}{*}{$\begin{array}{c}\text { Neurological } \\
\text { symptoms }\end{array}$} & \multicolumn{2}{|c|}{ Auto-antibodies profile } & \multirow{2}{*}{$\begin{array}{c}\text { State after } \\
\text { GFD }\end{array}$} \\
\hline & & Gluten p & Gluten - & \\
\hline 1 & Headache & Anti-sulfatide IgG & - & U \\
\hline 2 & Epilepsy & - & - & I \\
\hline 3 & Epilepsy and PN & - & - & l \\
\hline 4 & Epilepsy & Anti-sulfatide IgG & - & I \\
\hline 5 & Headache & - & Anti-sulfatide $\lg A$ & U \\
\hline 6 & Headache & - & & 1 \\
\hline
\end{tabular}

significance level was further verified by multiple-comparison analysis with Tukey's test. Categorical variables were compared by $v^{2}$ (chi square) or Fisher's exact tests.

\section{Results}

Twenty-two adult CD patients and twenty healthy subjects were enrolled (Table 1). The $11(50 \%)$ patients with a nonclassical CD picture, presented: iron deficiency anemia 7 (64\%), hypertransaminasemia 2 (18\%), osteoporosis $1(9 \%)$, recurrent abortions $2(18 \%)$ and neurological symptoms 3 (27\%). In particular, 6 patients reported neurological symptoms, 3 in a clinical picture compatible with a classical CD and 3 with a non-classical CD (4 females), mean age at diagnosis 38 (range 33-43) and mean GFD duration 36 months (range 12-84). Among them, four patients reported an improvement after a strict GFD (Table 2).

The auto-antibody profile of the enrolled $C D$ patients before and after GFD is reported in Table 3. On a gluten-containing diet, anti-sulfatide $\operatorname{IgA}$ antibodies were present in 3 (19\%) patients and only in one case their persistence was observed after a strict GFD. Moreover, anti-sulfatide IgA antibodies appeared in one case during GFD; no anti-sulfatide IgA positivity was observed in all 6 patients having neurological symptoms on a gluten-containing diet (Table 2).

Anti-sulfatide, anti-GM1 and anti-GM2 IgM antibodies were also observed in 2 treated patients. All the other autoantibodies were absent and no demographic or clinical parameters were associated with their positivity.

On a gluten-containing diet, anti-sulfatide IgG antibodies were observed in 8 (36\%) patients of our study. Among them, 2 patients presented neurological disorders (headache, epilepsy) with no statistical difference between the group of patients with and without neurological disorders. Anti-
Table 3. Patients included in the study and their auto-antibodies status before and after gluten-free diet. CD patients $\left(n^{1 / 4} 22\right)$ (1)

\begin{tabular}{lcc} 
Type of auto-antibody & $\begin{array}{c}\text { Number of positive } \\
\text { patients during } \\
\text { gluten-containing diet }\end{array}$ & $\begin{array}{c}\text { Number of positive } \\
\text { patients during } \\
\text { gluten-free diet }\end{array}$ \\
\hline IgG anti-sulfatide & 8 & 0 \\
IgG anti-ganglio side & 0 & 0 \\
IgA anti-sulfatide & 3 & 2 \\
IgA anti-ganglio side & 0 & 0 \\
IgM anti-sulfatide & 0 & 1 \\
IgM anti-ganglioside & 0 & 2
\end{tabular}

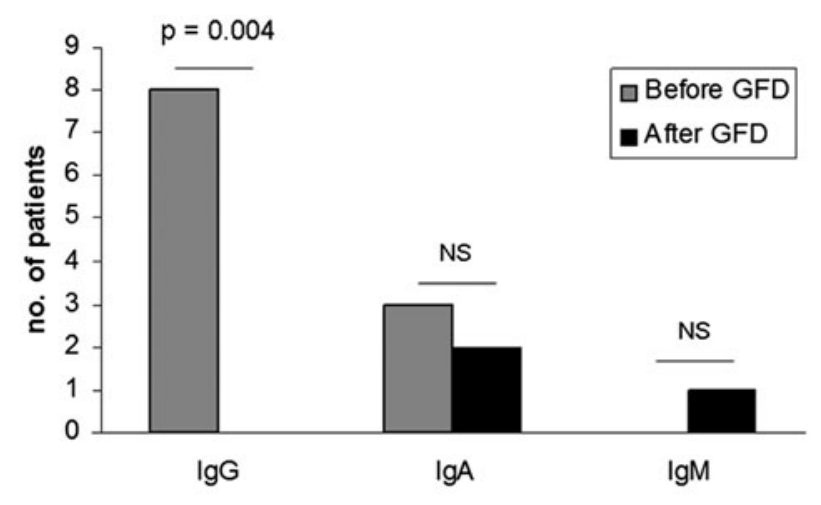

Anti-sulfatide antibodies
Figure 1. Anti-sultatıde IgG, IgA, IgM reactivity in cellac patients betore and after gluten-free diet. GFD: gluten-free diet; NS: not significant.

sulfatide IgG antibodies disappeared in all the patients during a GFD (Figure 1).

In general, 12 (59\%) CD patients presented at least one of the tested auto-antibodies vs. none of the non-CD controls presented them $(p<0.0001)$. While on a gluten-containing diet, $10(45 \%)$ of $C D$ patients presented at least one of the tested auto-antibodies vs. 4 (18\%) during GFD ( $\left.p^{1 / 4} 0.02\right)$.

\section{Discussion}

For the first time in $C D$, we found the presence of anti-sulfatide IgG and IgA auto-antibodies. Anti-sulfatide IgG disappeared during GFD, while IgA were independent of the gluten withdrawal (Figure 1).

The binding site and the fine specificity of the anti-sulfatide antibodies may determine a particular clinical neuropathic syndrome. The presence of anti-sulfatide IgG and IgA antibodies was reported in patients with neurological disorders. The anti-sulfatide IgG antibodies were initially found in patients with acute immune-mediated polyradiculoneuropathy, the Guillain-Barre Syndrome (GBS) while anti-sulfatide and anti-ganglioside IgA antibodies in patients affected by GBS, after Campylobacter jejuni enteritis.[16-19]

The gangliosides in peripheral nerves were subsequently targeted by serum antibodies in patients with GBS and interaction between anti-ganglioside IgG antibodies and peripheral nerve ganglioside is believed to cause GBS and its variants.[20]

More recently, high titers of anti-sulfatide antibodies, mostly IgM, have been associated with inflammatory demyelinating neuropathies [21] and subsequently with a chronic 
mostly sensorimotor dysimmune neuropathy $[16,9]$ and smallfiber neuropathy.[22,23]

Alaedini et al. [6] detected the presence of IgG antibodies against at least one or more gangliosides (GM1, GM2, GD1a and GD1b) in 6 adult CD patients with distal sensory neuropathy, suggesting a T-cell mediated autoimmune response against gangliosides. Volta et al. [12] found a positivity to at least one of the three anti-ganglioside IgG antibodies (GM1, GD1b and GQ1b) in 64\% of CD patients with a neurological dysfunction and in $30 \%$ of $C D$ patients without neurological symptoms. Although the limited number of patients precludes a definitive statement, no association has been found between the analyzed auto-antibodies and the neurological symptoms included headache,[24] as recently described in $C D$ in our study.

Anti-ganglioside IgA antibodies have been reported for the first time in patients affected by GBS.[18] In particular, anti-ganglioside GM1 IgA antibodies have been detected in patients with GBS after Campylobacter jejuni enteritis,[19] suggesting a possible link between intestinal inflammation, IgA production and autoimmune neuropathies. An autoimmune response to GM1 could be determined by molecular mimicry and a successive T-cell mediated pathway, responsible for the clinical manifestations. However, in line with our findings, no association has been reported between IgA and clinical phenotypes. In our study, the absence of anti-ganglioside and anti-sulfatide IgA antibodies in $C D$ patients with neurological symptoms on a gluten-containing diet may suggest a different mechanism while the presence of anti-sulfatide IgA antibodies during GFD may indicate higher susceptibility to develop an autoimmune response independently of gluten.

Anti-sulfatide, anti-GM1 and anti-GM2 IgM antibodies were also observed in 2 treated $C D$ patients without neurological disorders. Both patients did not show other autoimmune diseases and no neurological disorder associated to these auto-antibodies, such as sensory demyelinating polyneuropathy and multifocal motor neuropathy.[9,25]

Gangliosides are highly expressed on intestinal epithelial cells [26] and anti-ganglioside antibodies have been described in $C D$ with neurological symptoms.[6,10-12] The binding of gliadin to ganglioside on the intestinal epithelium might have a role in the anti-gliadin immune response and contribute to the intestinal inflammatory reaction in CD.[27]

The presence of IgG auto-antibodies in our patients with and without neurological symptoms on a gluten-containing diet and their total absence in non-CD controls suggests a possible connection between auto-antibodies and the enteropathy through a similar mechanism. Their disappearance in all the patients may be suggestive of a possible link between gluten and IgG auto-antibodies.

Anti-sulfatide IgG reactivity in patients with non-classical $\mathrm{CD}$ and neurological symptoms may indicate an acute phase of the neurological disease, but no acute neurological disorder has been reported with these auto-antibodies in our patients.

Further analyses with a larger patient group are needed to elucidate the possible association between anti-sulfatide antibodies and neurological manifestation of $C D$ and to elucidate their role, described here for the first time.

\section{Disclosure statement}

The authors report no conflicts of interest. The authors alone are responsible for the content and writing of this article.

\section{Funding}

Zentech and Alifax supported the study with the forniture of media, antibodies and kits.

\section{References}

[1] Wills A, Hovell CJ. Neurological complications of enteric disease. Gut. 1996;39:501-504.

[2] Kaplan JG, Pack D, Horoupian D, et al. Distal axonopathy associated with chronic gluten enteropathy: a treatable disorders. Neurology. 1988;38:642-645.

[3] Finelli PF, McEntee WJ, Ambler M, et al. Adult celiac disease presenting as cerebellar syndrome. Neurology. 1980;30:245-249.

[4] Gobbi G, Bouquet F, Greco L, et al. Coeliac disease, epilepsy, and cerebral calcifications. The Italian Working Group on Coeliac Disease and Epilepsy. Lancet. 1992;340:439-443.

[5] Diamanti A, Capriati T, Bizzarri C, et al. Autoimmune diseases and celiac disease which came first: genotype or gluten? Expert Rev Clin Immunol. 2016;12:67-77.

[6] Alaedini A, Green PH, Sander HW, et al. Ganglioside reactive antibodies in the neuropathy associated with celiac disease. J Neuroimmunol 2002;127:145-148.

[7] Willison HJ, Yuki N. Peripheral neuropathies and anti-glycolipid antibodies. Brain. 2002;125:2591-2625.

[8] Norton WT, Cammer W. Isolation and characterization of myelin. In: Morell P, editor. Myelin. 2nd ed. New York: Plenum; 1984. p. 174-178.

[9] Carpo M, Meucci N, Allaria S, et al. Anti-sulfatide IgM antibodies in peripheral neuropathy. J Neurol Sci. 2000;176:144-150.

[10] Briani C, Zara G, Alaedini A, et al. Neurological complications of celiac disease and autoimmune mechanisms: a prospective study. J Neuroimmunol. 2008;195:171-175.

[11] Tursi A, Giorgetti GM, lani C, et al. Peripheral neurological disturbances, autonomic dysfunction, and antineuronal antibodies in adult celiac disease before and after a gluten-free diet. Dig Dis Sci. 2006;51:1869-1874.

[12] Volta U, De Giorgio R, Granito A, et al. Anti-ganglioside antibodies in coeliac disease with neurological disorders. Dig Liver Dis. 2006;38:183-187.

[13] Oberhuber G, Granditsch G, Vogelsang H. The histopathology of coeliac disease: time for a standardized report scheme for pathologists. Eur J Gastroenterol Hepatol. 1999;11:1185-1194.

[14] Ludvigsson JF, Leffler DA, Bai JC, et al. The Oslo definitions for coeliac disease and related terms. Gut. 2013;62:43-52.

[15] Associazione Italiana di Neuroimmunologia. Diagnostica delle neuropatie periferiche disimmuni. In: Standardizzazione di procedimenti e metodiche laboratoristiche in neuro immunologia. September; Verona Italy: AINI; 2004. p. 21-26.

[16] Van den Berg LH, Lankamp CLAM, De Jager AEJ, et al. Anti-sulphatide antibodies in peripheral neuropathy. J Neurol Neurosurg Psychiatry. 1993;56:1164-1168.

[17] Ilyas AA, Mithen FA, Dalakas MC, et al. Antibodies to sulfated glycolipids in Guillain-Barre syndrome. J Neurol Sci. 1991;105:108-117.

[18] Ilyas AA, Mithen FA, Chen ZW, et al. Anti-GM1 IgA antibodies in Guillain-Barre syndrome. J Neuroimmunol. 1992;36:69-76.

[19] Koga M, Yuki N, Hirata K. Subclass distribution and the secretory component of serum IgA anti-ganglioside antibodies in GuillainBarre syndrome after Campylobacter jejuni enteritis. J Neuroimmunol. 1999;96:245-250.

[20] Claudie C, Vial C, Bancel J, et al. Antiganglioside autoantibody profiles in Guillain-Barre syndrome. Ann Biol Clin (Paris). 2002;60:589-597.

[21] Fredman P, Vedeler CA, Nyland $H$, et al. Antibodies in sera from patients with inflammatory demyelinating polyradiculoneuropathy 
react with ganglioside LM1 and sulphatide of peripheral nerve myelin. J Neurol. 1991;238:75-79.

[22] Pestronk A. Invited review: motor neuropathies, motor neuron disorders, and antiglycolipid antibodies. Muscle Nerve. 1991;14:927-936.

[23] Dabby R, Weimer LH, Hays AP, et al. Antisulfatide antibodies in neuropathy: clinical and electrophysiologic correlates. Neurology. 2000;54:1448-1452.

[24] Dimitrova AK, Ungaro RC, Lebwohl B, et al. Prevalence of migraine in patients with celiac disease and inflammatory bowel disease. Headache. 2013;53:344-355.
[25] Nobile-Orazio E. Multifocal motor neuropathy. J Neuroimmunol. 2001;115:4-18.

[26] Holgersson J, Str6mberg N, Breimer ME. Glycolipids of human large intestine: difference in glycolipid expression related to anatomical localization, epithelial/non-epithelial tissue and the $A B O$, Le and Se phenotypes of the donors. Biochimie. 1988;70: 15651574.

[27] Alaedini A, Latov N. Transglutaminase-independent binding of gliadin to intestinal brush border membrane and GM1 ganglioside. J Neuroimmunol. 2006;177:167-172. 